\title{
Pressure Variation of the Strain State of MnAs Nanoclusters Embedded in GaAs
}

\author{
J. Bak-Misiuk ${ }^{a}$, E. Dynowska ${ }^{a}$, P. Romanowski ${ }^{a}$, A. Misiuk ${ }^{b}$, J. SAdowski $^{a, c}$ And \\ W. CALIEBE $^{d}$ \\ ${ }^{a}$ Institute of Physics, Polish Academy of Sciences, al. Lotnikow 32/46, PL-02668 Warsaw, Poland \\ ${ }^{b}$ Institute of Electron Technology, al. Lotnikow 46, PL-02668 Warsaw, Poland \\ ${ }^{c}$ MAX-Lab, Lund University P.O. Box. 118, S-22100 Lund, Sweden \\ ${ }^{d}$ HASYLAB at DESY, Notkestr. 85, D-22603 Hamburg, Germany
}

\begin{abstract}
Granular GaAs:(Mn,Ga)As films were prepared by annealing at $500{ }^{\circ} \mathrm{C}$ under ambient and enhanced hydrostatic pressure $(1.1 \mathrm{GPa})$, of $\mathrm{Ga}_{1-x} \mathrm{Mn}_{x} \mathrm{As} / \mathrm{GaAs}$ layers $(x=0.025,0.03,0.04,0.05$ and 0.063$)$ grown at $230{ }^{\circ} \mathrm{C}$ by molecular beam epitaxy method. Distinct influence of enhanced hydrostatic pressure applied during sample annealing on strain state of inclusions was found. An increase of lattice distortion and of strain of inclusions for the samples treated under hydrostatic pressure is related to different bulk moduli of GaAs and of MnAs
\end{abstract}

PACS: 61.05.cp, 75.50.Pp, 81.40.Vw

\section{Introduction}

Rapid development of spintronics is observed in recent years. This exciting new field of research combines magnetism and electronics or optoelectronics. In the last decade, films built from ferromagnetic MnAs clusters embedded in GaAs lattice are very attractive since they show giant magneto-optical effects at room temperature. Such layers are frequently called "granular layers" and denoted GaAs:MnAs. It has been demonstrated that such layers are formed in effect of annealing at high temperatures (HT) of $\mathrm{Ga}_{1-x} \mathrm{Mn}_{x}$ As layers [1-8]. During annealing, layer decomposition occurs and MnAs or (Mn, Ga) As ferromagnetic precipitates are fairly easily produced. Depending on the annealing conditions and on Mn concentration, small GaMnAs cubic, or larger of the NiAs-type $\mathrm{MnAs}$ with the space group $\mathrm{P} 6_{3} / m m c$ [9], nanoclusters are created $[1,5-8]$. Typical sizes of the cubic clusters are of $2-3 \mathrm{~nm}$ for the layer with $2 \% \mathrm{Mn}$ content and they increase up to 3-4 $\mathrm{nm}$ in the layers with Mn concentration of up to $6 \%[6,8]$. Due to their small dimension they can not be detected by X-ray diffraction, but they are visible in electron microscopy studies $[6,8]$. Distorted, as compared to MnAs bulk [3, 8] hexagonal MnAs clusters with diameter up to $20 \mathrm{~nm}$ are formed during annealing at higher temperature $\left(T \geq 500{ }^{\circ} \mathrm{C}\right)$ of $\mathrm{Ga}_{1-x} \mathrm{Mn}_{x}$ As with higher Mn concentration.

Distortion of the hexagonal MnAs inclusions introduces compressive strain into the GaAs matrix [8]. Therefore the creation of granular GaAs:(Mn, Ga)As layers is accompanied with contraction of the lattice parameters of the GaAs matrix - its out-of-plane lattice parameter, measured in the direction perpendicular to the interface, is usually smaller than that of the GaAs substrate (see e.g. $[3,7,8]$ ).
As it was stated earlier, not only temperature, but also enhanced hydrostatic pressure (HP) applied during annealing of semiconductors changes diffusivity of interstitials Mn in GaMnAs and affects their structural and magnetic properties considerably [10, 11]. In effect of the HP-HT treatment and due to the differences in compressibility and thermal expansion of primary existing precipitates, some additional defects can nucleate and grow at the existing primary structural irregularities [12]. Distinct influence of hydrostatic pressure applied during annealing on layer strain has been found in $\mathrm{Ga}_{1-x} \mathrm{Mn}_{x} \mathrm{As} / \mathrm{GaAs}$ [13]. The HP-induced layer strain is definitely dependent on the Mn content. An increase of this strain was about three times higher, for the sample with the highest $\mathrm{Mn}$ concentration $\left(\mathrm{Ga}_{0.937} \mathrm{Mn}_{0.063} \mathrm{As}\right)$ containing after annealing mainly hexagonal inclusions in comparison to the sample with the lowest Mn concentration $\left(\mathrm{Ga}_{0.975} \mathrm{Mn}_{0.025} \mathrm{As}\right)$. This effect could be explained by pressure-dependent distortion of the hexagonal MnAs nanoclusters. Therefore, further studies were undertaken. In this work the results of these studies are presented.

\section{Experiment}

$\mathrm{Ga}_{1-x} \mathrm{Mn}_{x}$ As layers with nominal Mn content of $x=$ $0.025,0.03,0.04,0.05$ and 0.063 were grown by $\mathrm{MBE}$ method on the (001)-oriented GaAs substrates at $230^{\circ} \mathrm{C}$. The $\mathrm{Ga}_{1-x} \mathrm{Mn}_{x}$ As layers were subjected to annealing at $500{ }^{\circ} \mathrm{C}$ for $30 \mathrm{~min}$ in argon atmosphere under ambient pressure $\left(10^{5} \mathrm{~Pa}\right)$ or under pressure of $1.1 \mathrm{GPa}$. Results of electron microscopy investigations of these samples, as-grown and annealed under ambient pressure at $500^{\circ} \mathrm{C}$, were presented in [8]. The HP-HT treatment was performed in specially designed high temperature-pressure 
furnace described earlier in detail [14]. The granular GaAs:(Mn, Ga)As layers were produced in this way.

Determination of the lattice parameters of hexagonal NiAs-type MnAs nanoclusters was performed using synchrotron radiation $(\lambda=1.54056 \AA)$ at the $\mathrm{W} 1$ beamline at DESY-Hasylab. The orientation of the MnAs nanoclusters embedded in GaAs matrix is well-defined the $\langle 00.1\rangle$ planes of MnAs are parallel to $\{111\} \mathrm{GaAs}$ planes. Therefore, the lattice parameters of MnAs were calculated from the 20.2 and 30.0 reflections detected by the $\omega$ scan in the vicinity of symmetrical $004 \mathrm{GaAs}$ reflection, and from the $2 \theta / \omega$ scan around the asymmetrical $224 \mathrm{GaAs}$ reflection, respectively. Detailed description of this procedure is given in $[3,8]$.

\section{Results and discussion}

An evidence of the presence of hexagonal nanoclusters

The crystal structure of the bulk MnAs is hexagonal of NiAs-type with lattice parameters $a=3.7187 \AA$ and $c=5.7024 \AA[15]$. So, the unit cells of the inclusions are distorted. This distortion creates the biaxial strain in the $\langle 00.1\rangle$ planes, $\varepsilon_{a}=\left(a_{\text {exp }}-a_{\text {bulk }}\right) / a_{\text {bulk }}$ and along the $c$-axis, $\varepsilon_{c}=\left(c_{\exp }-c_{\text {bulk }}\right) / c_{\text {bulk }}$. The values of strain for the $\mathrm{Ga}_{1-x} \mathrm{Mn}_{x}$ As layers $(x \geq 0.04)$ annealed under ambient pressure at $500{ }^{\circ} \mathrm{C}$ are: $\varepsilon_{a}=-2 \times 10^{-3}$, and $\varepsilon_{c}=19 \times 10^{-3}$. These values are very close to those published earlier [3]. For the samples annealed under HP the lattice parameters of inclusions and values of strain increased. The average strain value in the $\langle 00.1\rangle$ planes for the HP-HT treated samples, $\varepsilon_{a \mathrm{HP}-\mathrm{HT}}=-4 \times 10^{-3}$ which is higher than that for the non HP treated samples, but still is relatively small. On the other hand, the strain along the $c$-axis after the $\mathrm{HP}-\mathrm{HT}$ treatment, $\varepsilon_{c \mathrm{HP}-\mathrm{HT}}$, is equal to $58 \times 10^{-3}$ and $32 \times 10^{-3}$ depending on the samples. The difference between the $c$ lattice parameters of MnAs inclusions as well as the related strain, $\varepsilon_{c \mathrm{HP}-\mathrm{HT}}$, can be caused by different defect structure of the inclusions [3]. The strain within inclusions, formed during annealing under ambient pressure, develops from both the lattice mismatch of $\mathrm{MnAs}$ and GaAs and large difference between the thermal expansion coefficients for these compounds (thermal strain) [3]. An increase of the lattice distortion and strain of inclusions, for the samples treated under HP, is related also to the different bulk moduli of GaAs matrix $\left(B_{M}=76 \mathrm{GPa}\right)$ and MnAs inclusions $\left(B_{I}=27 \mathrm{GPa}\right)$. During the HP treatment, the difference in compressibility of the MnAs inclusions $\left(1 / B_{I}\right)$ and of the GaAs matrix $\left(1 / B_{M}\right)$ involves additional internal strain according to the equation:

$$
\varepsilon_{\mathrm{HP}}=\frac{\mathrm{HP}}{3}\left(\frac{1}{B_{I}}-\frac{1}{B_{M}}\right) .
$$

In the case of our materials pressure-induced strain, $\varepsilon_{c}$, within the inclusions should be a tensile one, being confirmed by our measurements (see Table).

From the knowledge of strains of the nanoclusters, the related stresses, $\delta_{a \mathrm{HT}}, \delta_{a \mathrm{HP}-\mathrm{HT}}, \delta_{c \mathrm{HT}}$ and $\delta_{c \mathrm{HP}-\mathrm{HT}}$ can be estimated through the Hooke's law using the elas- was enabled owing to the use of synchrotron radiation. Hexagonal nanoclusters were detected only for the samples with Mn concentration $x>0.03$ [8] for which the HP-HT treatment caused a pronounced change of the layer strain [13]. In the case of layers with smaller Mn concentration, annealed at higher temperatures, formation of cubic nanoclusters is still preferable [8]. The $\omega$ scan recorded around the $004 \mathrm{GaAs}$ reflection allowed the detection of the two 20.2 reflections originating from the hexagonal NiAs-type MnAs nanoclusters [3, 8].

From the $2 \theta / \omega$ scans of the $20.2 \mathrm{MnAs}$ reflection and around the $22.4 \mathrm{GaAs}$ asymmetrical one (Fig. 1a, b) the lattice spacing $d_{20.2}$ and $d_{30.0}$ were calculated. On the basis of these values the $a$ and $c$ lattice parameters of the hexagonal unit cell of MnAs clusters, as well as the strain values were determined. The obtained results are given in the Table.
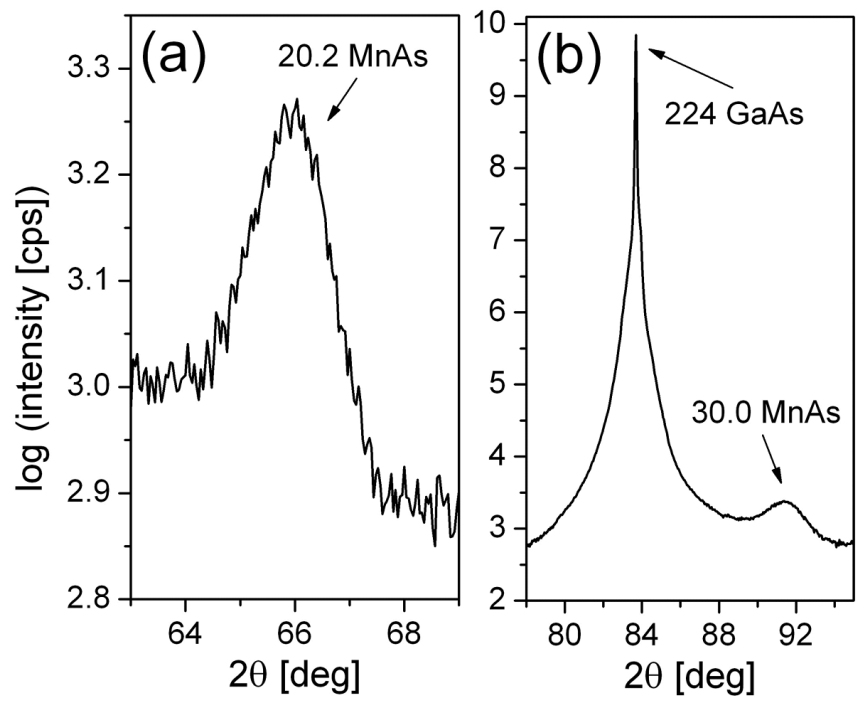

Fig. 1. The X-ray patterns of $\mathrm{Ga}_{0.937} \mathrm{Mn}_{0.063} \mathrm{As}$ after annealing at $500{ }^{\circ} \mathrm{C}$ under $1.1 \mathrm{GPa}$. (a) - the $2 \theta / \omega$ scan of $20.2 \mathrm{MnAs}$ reflection; (b) - the $2 \theta / \omega$ scan of $30.0 \mathrm{MnAs}$ reflection.

tic constants for hexagonal MnAs [16]. These stresses are following: $\delta_{a \mathrm{HT}}=-0.04 \mathrm{GPa}, \delta_{c \mathrm{HT}}=2 \mathrm{GPa}$, $\delta_{a \mathrm{HP}-\mathrm{HT}}=-0.08$ and $\delta_{c \mathrm{HP}-\mathrm{HT}}=3.7 \mathrm{GPa}$ or $6.3 \mathrm{GPa}$ (depending on the sample). Let us notice that the compressive stress acting in the (00.1) plane, $\delta_{a}$, of inclusions is much smaller than the tensile stress, $\delta_{c}$, acting out of the (00.1) plane. Therefore, in further considerations we will take into account only the $\delta_{c}$ stress. The same stress, but with an opposite sign, acts in $\langle 111\rangle$ directions of the GaAs matrix unit cell. It means that GaAs matrix is hydrostatically compressed [8] and the unit cell compression increases for HP-HT treated samples $\left(\delta_{c \mathrm{HP}-\mathrm{HT}}>\delta_{c \mathrm{HT}}\right)$. In this way an influence of hydrostatic pressure applied during annealing on strain of GaAs matrix contained MnAs hexagonal inclusions was explained. For samples with lower Mn concentration con- 
TABLE

Lattice parameters and strains of the hexagonal MnAs inclusions produced in $\mathrm{Ga}_{1-x} \mathrm{Mn}_{x}$ As layers after HT and HP-HT annealing

\begin{tabular}{l|c|c|c|c|c|c|c|c}
\hline \hline Layer & \multicolumn{4}{|c}{ After HT annealing } & \multicolumn{3}{c}{ After HP-HT annealing } \\
\hline$x$ & $\begin{array}{c}a[\AA] \\
\pm 0.002\end{array}$ & $\begin{array}{c}\varepsilon_{a \mathrm{HT}} \\
\pm 5 \times 10^{-4}\end{array}$ & $\begin{array}{c}c[\AA] \\
\pm 0.007\end{array}$ & $\begin{array}{c}\varepsilon_{c \mathrm{HT}} \\
\pm 1 \times 10^{-3}\end{array}$ & $\begin{array}{c}a[\AA] \\
\pm 0.002\end{array}$ & $\begin{array}{c}\varepsilon_{a \mathrm{HP}-\mathrm{HT}} \\
\pm 5 \times 10^{-4}\end{array}$ & $\begin{array}{c}c[\AA] \\
\pm 0.007\end{array}$ & $\begin{array}{c}\varepsilon_{c \mathrm{HP}-\mathrm{HT}} \\
\pm 1 \times 10^{-3}\end{array}$ \\
\hline 0.04 & 3.711 & $-2 \times 10^{-3}$ & 5.812 & $19 \times 10^{-3}$ & 3.734 & $-4 \times 10^{-3}$ & 6.03 & $58 \times 10^{-3}$ \\
\hline 0.05 & 3.710 & $-2 \times 10^{-3}$ & 5.812 & $19 \times 10^{-3}$ & 3.734 & $-4 \times 10^{-3}$ & 5.884 & $32 \times 10^{-3}$ \\
\hline 0.063 & 3.709 & $-2 \times 10^{-3}$ & 5.812 & $19 \times 10^{-3}$ & 3.727 & $-4 \times 10^{-3}$ & 5.884 & $32 \times 10^{-3}$
\end{tabular}

taining mainly cubic MnAs, small influence of pressure applied during annealing on strain state of the matrix is probably caused by small differences between the bulk moduli of GaAs and cubic MnAs.

\section{Conclusions}

Enhanced hydrostatic pressure applied during annealing at $500{ }^{\circ} \mathrm{C}$ of the MBE-grown GaMnAs layers results in creation of increasingly strained MnAs inclusions. The strain induced by this procedure is definitely dependent on the crystallographic structure of MnAs clusters (cubic or hexagonal) and it is much higher for the hexagonal ones. In consequence, the annealing under pressure leads to the increase of the strain of the GaAs matrix, especially for the layers containing the hexagonal MnAs inclusions.

\section{References}

[1] M. Moreno, A. Trampert, B. Jenichen, L. Däweritz, K.H. Ploog, J. Appl. Phys. 92, 4672 (2002).

[2] M. Moreno, B. Jenichen, V.M. Kaganer, W. Braun, L.A. Trampert, L. Däweritz, K.H. Ploog, Phys. Rev. $B$ 67, 235206 (2003).

[3] M. Moreno, V.M. Kaganer, B. Jenichen, A. Trampert, L. Däweritz, K.H. Ploog, Phys. Rev. B 72, 15206 (2005).

[4] M. Moreno, B. Jenichen, L. Däweritz, K.H. Ploog, Appl. Phys. Lett. 86, 161903 (2005).

[5] M. Yokoyama, H. Yamaguchi, T. Ogawa, M. Tanaka, J. Appl. Phys. 97, 10D317 (2005).

[6] A. Kwiatkowski, D. Wasik, M. Kaminska, R. Bozek, J. Szczytko, A. Twardowski, J. Borysiuk, J. Sadowski, J. Gosk, J. Appl. Phys. 101, 113912 (2007).
[7] I.N. Demchenko, K. Lawniczak-Jablonska, T. Story, V. Osinniy, R. Jakiela, J.Z. Domagala, J. Sadowski, M. Klepka, A. Wolska, M. Chernyshova,J. Phys.: Condens. Matter 19, 496205 (2007).

[8] K. Ławniczak-Jabłońska, J. Bak-Misiuk, E. Dynowska, P. Romanowski, J.Z. Domagała, J. Libera, A. Wolska, M. Klepka, P. Dłużewski, J. Sadowski, A. Barcz, D. Wasik, A. Twardowski, A. Kwiatkowski, J. Solid State Chemistry 184, 1530 (2011).

[9] JCPDS 28-0664.

[10] A. Misiuk, J. Bak-Misiuk, B. Surma, W. Osinniy, M. Szot, T. Story, J. Jagielski, J. Alloys Comp. 423, 201 (2006).

[11] J. Bak-Misiuk, J.Z. Domagala, E. Dynowska, E. Lusakowska, A. Misiuk, W. Paszkowicz, J. Sadowski, A. Barcz, W. Caliebe, Rad. Phys. Chem. 78, S116 (2009).

[12] J. Jung, Philos. Mag. A 50, 257 (1984).

[13] J. Bak-Misiuk, P. Romanowski, A. Misiuk, J. Sadowski, R. Jakiela, A. Barcz, accepted for publication in J. Nanoscience and Nanotechnology.

[14] A. Misiuk, Mater. Phys. Mech. 1, 119 (2000).

[15] J. Mira, F. Rivadulla, J. Rivas, A. Fondado, T. Guidi, R. Caciuffo, F. Carsughi, P.G. Radaelli, J.B. Goodenough, Phys. Rev. Lett. 90, 097203 (2003).

[16] M. Dörfer, K. Bärner, Phys. Stat. Sol. A17, 141 (1973). 